\title{
FORMACIÓN DEL PROFESORADO DE PEDAGOGÍA EN EL ENFOQUE INTERCULTURAL UNIVERSIDAD VERACRUZANA-MÉXICO
}

\author{
TRAINING OF TEACHERS OF PEDAGOGY IN THE INTERCULTURAL \\ APPROACH UNIVERSIDAD VERACRUZANA-MEXICO
}

\author{
María Guadalupe Ñeco Reyna*
}

\begin{abstract}
El artículo aborda la temática de que la Universidad Veracruzana como institución de educación superior debe incorporar a sus agendas institucionales, entre otros temas de interés público, el relacionado con la formación del profesorado en el enfoque educativo intercultural, toda vez que es un espacio privilegiado de formación desde donde se pueden reproducir de manera exponencial conocimientos, habilidades, valores y actitudes que favorezcan el reconocimiento de la diverdad entre los hombres y las mujeres. El método utilizado es el denominado hermenéutico interpretativo, los resultados revelan que el profesorado de pedagogía valora lo intercultural en el aspecto teórico y práctico, que existe interés por comprender el enfoque intercultural e identifican necesidades al respecto en su formación profesional. Reconocen que hace falta incorporar en su vida institucional acciones que reconstruyan sus planes y programas de estudio y resignifiquen contenidos en el campo de lo intercultural. En tal sentido, como interés fundamental para la sociedad en general y, por esta razón, para las distintas instituciones educativas del estado de Veracruz y del país.

Palabras claves: Profesorado universitario, actitudes, habilidades, valores, enfoque intercultural.
\end{abstract}

The article addresses the theme that the Universidad Veracruzana as an institution of higher education should incorporate into its institutional agendas, among other topics of public interest, the one related to teacher training in the intercultural educational approach, since it is a privileged space of formation from which knowledge, abilities, values and attitudes that favor the recognition of the diversity between men and women can be exponentially reproduced. The method used is the so-called interpretive hermeneutics, the results reveal that teachers of pedagogy, values the intercultural in the theoretical and practical aspect, that there is interest in understanding the intercultural approach and identify needs in their professional training. They recognize that it is necessary to incorporate into their institutional life, actions that reconstruct their plans and programs of study and redefine contents in the field of intercultural. In this sense, as a fundamental interest for society in general and therefore for the different educational institutions of the State of Veracruz and the country.

Key words: University teachers, attitudes, beliefs, values, intercultural approach.

\section{Introducción}

Los retos que se avizoran para el siglo XXI deben sumar, entre sus prioridades, el educar en y para vivir en sociedades multiculturales, que demandan visibilizar la diversidad cultural del contexto socioeducativo, para comprender, transformar y actualizar la práctica docente. Un hecho innegable es la globalización, que plantea la necesidad de diseñar políticas públicas, enfocadas a impulsar y fortalecer la perspectiva intercultural en el ámbito educativo e implementar acciones pedagógicas, que permitan incidir en la formación del profesorado universitario respecto del enfoque intercultural y cómo se cristaliza en los espacios educativos, atendiendo con esto uno de los muchos pendientes de la agenda educativa que se tienen en México y que repetidamente, se dice, tienen como fuente su heterogeneidad cultural.

El debate de la globalización se manifiesta en la dimensión económica, política y por supuesto impactan la educativa y pone en evidencia cómo nuestras sociedades se ven afectadas por parecidas influencias de préstamo, cambio e interacción cultural y a menudo estas desembocan en procesos de uniformización cultural (Berger, 2002; Gimeno, 2002; Warnier, 2002; Verdú, 2003).

Ante estas complejas circunstancias socioculturales es fundamental colocar en el centro del análisis la formación del profesorado universitario atendiendo con esto uno de los muchos pendientes que se viven en México y que repetidamente, se dice, tienen como fuente su heterogeneidad cultural. Inscribiendo con esto una tendencia reformadora en la praxis educativa,

\footnotetext{
* Universidad Nacional de Educación a Distancia (UNED), Madrid, España. Correo electrónico: niecoreynama@ hotmail.com
} 
que trate de responder a la diversidad cultural de las sociedades actuales (Aguado, 2009).

A nivel internacional las voces de los expertos demandan re-pensar la formación del profesorado ante sociedades complejas y cambiantes, mover a la reflexión y acción de las respuestas pedagógicas que la sociedad demanda y representan retos y desafíos en el tejido sociocultural construido. Para comprender las formas de mirar, entender el mundo y reaprender las formas de producción y aplicación del conocimiento. Señala Bello y Cols. (2016) "[...] el argumento se encuentra en los indicadores de Desarrollo Humano y de los Objetivos de Desarrollo del Milenio que reportan avances para la región, pero se mantienen las brechas que manifiestan grandes desigualdades" (p. 37).

Mientras que en América Latina la problemática que genera la reflexión acerca de la interculturalidad tiene un pasado enraizado en el colonialismo, ya que está estrechamente vinculada con las luchas de los pueblos indígenas de toda la región, inclusive, la significación de la interculturalidad presenta variantes según las características y luchas sociales de las distintas regiones latinoamericanas.

El grupo "Modernidad/colonialidad" que conforman autores intelectuales, presentan un carácter heterogéneo y multidisciplinar, sobre todo coinciden con el pensamiento de Boaventura de Sousa Santos. La perspectiva que asumen puede caracterizarse como una construcción alternativa a la modernidad eurocéntrica, tanto en su proyecto de civilización como en sus propuestas epistémicas. Especialistas en el tema de la Interculturalidad critican respecto de cómo se mira desde Latinoamérica lo intercultural en Educación, algunos de ellos son; el argentino Enrique Dussel, desde Perú Aníbal Quijano, y el argentino norteamericano Walter Mignolo, los puertorriqueños Ramón Grosfoguel y Nerlson Maldonado Torres y el colombiano Arturo Escobar.

Asimismo, desde el Ecuador afirma Walsh (2005) que con frecuencia se confunde el enfoque intercultural con un enfoque cultural o multicultural, siendo que la interculturalidad debiera ser considerada menos como sustantivo y más como verbo de acción, tarea de toda sociedad y no directamente en sectores indígenas y afrodescendientes.

Siguiendo con estas opiniones, en México en muchas ocasiones la política en educación omite lo intercultural y se rige por cuestiones económicas y de mercado, en la llamada erróneamente reforma educativa, ya que obedece más a un carácter de reforma laboral, imponiendo modelos educativos con enfoques por competencias, que en muchos de los casos tienden a una mirada empresarial.

En aras y con el ánimo de enfatizar los hallazgos y aportes teóricos de estudios interculturales hechos con antelación, se analiza el presentado en la Universidad Complutense de Madrid (Alonso, 2006), quien trata de responder ¿qué es la educación intercultural? Este cuestionamiento tiene relación directa con nuestra temástica, se describe la educación intercultural como un enfoque configurador de un proceso educativo, en orden a lograr una comunicación humana plena, dentro de una sociedad que ha llegado a ser intercultural. Para ello se detiene en resaltar que la sociedad actual es multicultural, condición inherente a nuestras sociedades actuales que no debemos perder de vista.

Un aporte teórico más de esta investigación, cuando enfatiza que la educación debe verse como factor de transformación social por medio de la educación intercultural. Otro hallazgo y coincidencia importante al afirmar que la educación intercultural se caracteriza por el intercambio, la interacción y favorece el desarrollo personal humano, para ello se deben adquirir competencias interculturales. Otra aportación teórica es la que realizó Gómez (2009), donde enfatiza la necesidad de fortalecer las competencias interculturales de los instructores comunitarios del Consejo Nacional de Fomento Educativo (CONAFE), que brindan su servicio en la Modalidad de Atención Educativa a Población Indígena (MAEPI), en San Cristóbal de las Casas, Chiapas (México).

Otra investigación que sirve de antecedente para fundamentar esta temática son los resultados del proyecto TALIS, realizado por la OCDE (2009), un estudio internacional pertinente a la enseñanzaaprendizaje, donde se reconoce esta necesidad universal de formación de lo intercultural, resultado de la presencia de poblaciones minoritarias tanto originarios como migrantes, en muchos países. Se indica con claridad la urgencia de trabajar en la formación de los futuros profesionales de la educación, en prácticas pedagógicas interculturales, con el fin de ir creando las condiciones para evitar conflictos, desde planos de igualdad, donde se respete la alteridad.

Así, estos trabajos contribuyen al campo de los estudios interculturales y reafirman la necesidad de estudios como el que se muestra, respecto de la urgente necesidad de generar acciones de formación del profesorado universitario respecto del enfoque educativo intercultural en la Educación Superior. 
Ante este panorama la problemática detectada en la formación del profesorado de la facultad de pedagogía, identificado mediante la observación directa y opiniones del estudiantado, se da sustento y origen a la pregunta de investigación.

¿Cuáles son los valores, creencias y actitudes interculturales que el profesorado universitario reconoce en su formación y si las aplica en su práctica docente, de la Facultad de Pedagogía de la Universidad Veracruzana?

Esta se plantea con la finalidad de identificar las acciones de la Universidad Veracruzana, una institución de educación superior de carácter público, que debe incorporar a sus agendas institucionales, entre otros temas, el relacionado con la formación del profesorado en el enfoque educativo intercultural, toda vez que es un espacio privilegiado de formación desde el que se pueden reproducir de manera exponencial conocimientos, habilidades, valores y actitudes que favorezcan el reconocimiento de la diverdad entre los hombres y las mujeres.

El sentido del artículo es pensar el enfoque intercultural en la formación del profesorado como algo necesario e importante, porque permite una mirada retrospectiva para reflexionar, analizar, comprender las formas de gestión de la diversidad cultural en la Universidad Veracruzana, y recuperar las respuestas pedagógicas que se dan ante la diversidad: social, política, género, sexualidad, etc. $\mathrm{El}$ análisis fue estrictamente descriptivo, ya que se intenta ofrecer un acercamiento a la importancia que la universidad concede al ámbito de la interculturalidad en el marco de una sociedad cada vez más pluricultural.

En consecuencia y atendiendo a Dietz (2012), el reconocimiento de la diversidad cultural, el desarrollo de programas educativos culturalmente pertinentes y la interculturalidad debe ser una forma de entablar relaciones entre grupos cultural, lingüística y étnicamente diversos. Esta recomendación exhorta precisar el concepto de cultura como esencial para el desarrollo humano, debido a que es un componente clave para conformar la identidad y preservar la memoria. En toda sociedad la cultura se convierte en elemento fundamental en el origen y dinámica de sus estructuras y valores. Analizar el enfoque intercultural como hermenéutica de las prácticas pedagógicas del profesorado hace eco con los principios asumidos por las sociedades que defienden los derechos de igualdad, democracia y participación social que conjuntamente son: la garantía que permite alcanzar los objetivos que dan sentido a la escuela, construcción de la identidad y el logro de la igualdad.

En afinidad con Aguado (2009) menciona la reflexión respecto de la educación, entendida como elaboración cultural y basada en la valoración de la diversidad cultural. "Promueve prácticas educativas dirigidas a todos y cada uno de los miembros de la sociedad en su conjunto" (p. 63). Por tanto, para que la educación sea realmente intercultural, es preciso que todas las culturas implicadas en el proceso educativo se den a conocer y se difundan en términos equitativos, incluyendo a todo el magisterio; contemplando en los contenidos curriculares los múltiples elementos de conocimiento y sabiduría de las diferentes culturas.

Siguiendo a Aguado Odina, Gil-Jaurena y Mata Benito (2005), defienden que la escuela debe atender a las diferencias culturales de los estudiantes, tanto por coherencia con los principios de la sociedad democrática y porque es la garantía para alcanzar objetivos educativos esenciales. En este tenor, Abdallah-Pretceille (2001) señala que las prácticas pedagógicas se desarrollan siguiendo varias concepciones teóricas y prácticas del profesorado; dentro de estas se puede, según lo intercultural, como hermenéutica planteada por esta autora, determinar la presencia de un enfoque intercultural.

Por consiguiente, en este marco comprensivo se propuso el objetivo; analizar valores, creencias $y$ actitudes interculturales que el profesorado universitario reconoce en su formación y si lo reconocen en su práctica profesional en la Facultad de Pedagogía de la Universidad Veracruzana, en Xalapa, Veracruz. Esto permite instaurar el escenario donde confluyen: la docencia reflexiva, respeto, diálogo y la convivencia armónica en un espacio formativo de interacción, favoreciendo la comprensión de la diversidad humana y el desarrollo de la competencia comunicativa intercultural, en el contexto de las sociedades presentes, entendiendo su pluralidad y su riqueza de expresiones.

La Universidad Veracruzana es una institución de educación superior, de carácter público, por esta razón está obligada a incorporar a sus agendas institucionales, entre otros temas de interés público, el relacionado con la formación del profesorado en el enfoque educativo intercultural, toda vez que es un espacio privilegiado de formación desde donde se pueden reproducir de manera exponencial conocimientos, habilidades, valores y actitudes que 
favorezcan el reconocimiento de la diversidad entre los hombres y las mujeres.

Esta acción propiciaría generar sistemas educativos que correspondan con las realidades y necesidades culturales de la población destinataria, fortalecidos con " $[. .$.$] esfuerzos de las instituciones$ de educación superior y han de ir acompañados por programas de diversificación tanto de los contenidos curriculares como de los métodos de enseñanzaaprendizaje" (Dietz 2008, p. 1).

Es así como en este artículo se enfoca la atención en la formación del profesorado universitario, quienes son los garantes de concretar el tipo de educación que recupere la diversidad como riqueza al interior de las aulas y los distintos espacios educativos. Por tanto, el reconocimiento de actitudes, habilidades y valores respecto del enfoque educativo intercultural del profesorado es un primer paso, que daría elementos que deriven en una reorientación de la forma en cómo se identifica y se trata pedagógicamente a la diversidad étnica, cultural, lingüística, de género, capacidades, talentos y estilos de aprendizaje de nuestros estudiantes.

Sin embargo, señala Galeano (2005), “[...] la diversidad cultural se percibe como un problema difícil de manejar y abordar", por tanto, se considera la opción de la negación de la situación, lo que no se $v e$, no se conoce ni se atiende, creyendo de manera errada que la diversidad es algo problemático. Sin embargo, el planteamiento y la apuesta práctica de una educación para el futuro ha de asumir el reto de educar en y para la interculturalidad.

En suma, el enfoque educativo intercultural es holístico y tiene un carácter inclusivo, parte del respeto y la valoración de la diversidad; es indispensable para lograr una educación integral, buscar erradicar elementos que dificulten la convivencia entre culturas como: discriminación, exclusión, racismo. La concepción de escuela para el enfoque intercultural es un espacio privilegiado para el desarrollo integral de las personas, el aprendizaje de la convivencia y la libertad solidaria, y la transformación social según principios de justicia social (Gorski, 2000).

Ante la pregunta ineludible ¿Por qué será importante la formación del profesorado en el enfoque intercultural? La respuesta inmediata es porque agrega valor a la práctica docente para promover y fomentar en las aulas la igualdad, democracia, respeto a la lengua materna, democracia y el equilibrio en relación con el contexto.
Ciertamente la igualdad es un ideal y una aspiración ética, que nunca implica homogeneizar, como señala Coleman (1990): "Somos conscientes de que no es posible lograr una completa igualdad de oportunidades, pero sí luchar por incrementarla, favorecerla y extenderla" (p. 29). Sin embargo, sería una falsa ilusión creer que la formación del profesorado universitario en el enfoque intercultural vendría a resolver todas las problemáticas en la comunidad universitaria. Siguiendo a Gimeno y cols. (1998, p. 114), mencionan: "No se explica solamente por la actuación autónoma de los profesores, sino que está ligada a aspectos culturales, institucionales, organizativos, curriculares, existencia de medios didácticos, etc., que no dependen del profesorado o no dependen del todo del profesor".

Se comprende que alinear las prácticas pedagógicas hacia los fines educativos quedó visible en la politización de los indicadores sociales y la reducción del conflicto social. "La percepción de la alteridad es, a la vez, producto y productora de identidad" (Dietz y Mateos, 2009, p. 50). Esta afirmación debiera mover los focos de atención y consideración del Sistema Educativo Mexicano (SEM), para desarrollar y promover el enfoque intercultural en la formación del profesorado, siendo la base de la formación humana.

Siguiendo a Fernández Enguita (1999), conocer el pensamiento y preconcepciones educativas que mantienen los docentes es un primer paso para entender cómo se puede cambiar la escuela y cómo puede influir el profesorado como filtro para desarrollar innovaciones y reformas educativas. Incluir la interculturalidad como eje transversal de la educación implica asumir la diversidad cultural desde una perspectiva de respeto y equidad social.

\section{Metodología}

El diseño metodológico de esta investigación cuenta con un enfoque cualitativo y al respecto Meneses (2004) indica " [...] la ciencia social es fruto del conocimiento conseguido y aceptado por el hombre por medio de procesos de reflexión, sistematización y rigor realizados con la finalidad de interpretar y comprender la realidad" (p. 224), en concordancia con el trabajo que se desarrolló, el que dio lugar al procedimiento de análisis.

Para interpretar la información se recurrió a la utilización de la técnica de análisis de contenido, asumiendo que los procesos de significación son 
construidos en la interacción humana, tratados como textos densos con significados, "que es atribuido por los mismos participantes del hecho educativo, Ricoeur como Gadamer valoran la importancia que tiene el contexto social" (Miguélez 2011, p. 45).

Estos autores coinciden con Dilthey (1948), quien hace hincapié en la importancia de la imaginación, gracias a ella, eleva lo causal al rango de lo significativo y representa lo típico como base de lo individual; el eslabón de enlace entre la Filosofía y las Ciencias Históricas forma la teoría de la interpretación o hermenéutica. Señala Navarro (2007) acerca de la evolución del análisis de contenido que se ha orientado hacia los tratamientos del material cualitativo situándolo en el ámbito de la metodología cualitativa. Siendo esta una de las grandes aportaciones de Glaser y Strauss (1967) citado en Valles (2000) respecto del desarrollo de la Grounded Theory, o "teoría enraizada" o "fundamentada", el procedimiento utilizado por esta teoría se denomina método comparativo constante y consiste en fundamentar la categorización y los análisis, que realiza en los mensajes que sobre la conducta de los actores sociales ofrece el texto en la experiencia y el bagaje teórico del propio investigador.

Para ello el proceso metodológico se dividió en varias etapas:

La primera se inició con el trabajo de los "actores sociales", es decir, todos aquellos informantes claves que de alguna u otra manera se pudieran contactar y llevar a cabo las entrevistas, para este caso me refiero a las y los coordinadoras de las áreas de conocimiento, que responden a la estructura organizacional de la Facultad de Pedagogía Sistema Escolarizado, escenario de la investigación. A este segmento de ocho personas se les aplicó entrevistas semiestructuradas, las que se fueron modificando a merced al trabajo de campo.

La segunda etapa se trabajó con dos grupos de estudiantes que pertenecían a dos experiencias educativas, impartidas por diferentes catedráticas de la facultad; Educación en Valores y Experiencia Recepcional, el criterio fue que llevaran un avance significativo en su trayectoria escolar y como alumnos de la facultad; con ellos se trabajaron dos grupos focales con la finalidad de recuperar sus opiniones respecto de lo intercultural y si lo reconocían en las prácticas pedagógicas de sus profesores y profesoras al trabajarlo en el contexto educativo.

Como se mencionó, el análisis de contenido está basado en la Teoría Fundamentada de Strauss y Glaser en Soneira (2012); la recuperación de la información se obtuvo mediante entrevistas y grupos focales, formalizadas en la intervención con los actores sociales que participaron en esta investigación.

Sugerido por Hernández (2012), se precisa que el análisis categorial y su relación con los informantes claves se fueron construyendo por medio de las redes conceptuales del tema que se abordó, combinando con la ayuda de la herramienta computacional del Atlas.ti.

\section{Resultados}

Para detectar cómo responden las y los profesores de la facultad de Pedagogía del Sistema Escolarizado, todos coordinadores y coordinadoras de las ocho áreas de conocimiento en las que se encuentra organizado el Plan de Estudios de Pedagogía (2000) acudimos a la recogida y análisis de la información, buscando la relación entre las habilidades, valores y actitudes interculturales que identifica el profesorado universitario en su formación profesional y si lo reconocen o no en su práctica docente. Entendiendo los valores como principios morales, ideológicos o de otro tipo que guían el comportamiento personal, y las habilidades como el conjunto de ideas que se tienen respecto de algo.

El análisis que se realizó fue exclusivamente descriptivo, ya que solo pretende ofrecer una primera aproximación a la importancia que la universidad veracruzana concede al ámbito de la interculturalidad en la formación del profesorado, en el marco de una sociedad cada vez más pluricultural. En el afán de alcanzar el objetivo de la investigación, se analizan los valores, creencias y actitudes interculturales que el profesorado universitario reconoce en su formación y si las reconocen en su práctica docente, a continuación se presentan los resultados obtenidos.

En la Tabla 1 se muestra información obtenida de los actores educativos que participaron en esta investigación, se decide colocar la información de esta manera para tener en un primer plano los datos.

Al observar las respuestas de las y los maestros se detecta la necesidad de querer informarse en cuanto al enfoque intercultural, ya que reconocen que trabajan con valores y desde el principio lo señalan en los grupos, pero las respuestas de los alumnos indican precisamente que solo se queda en el discurso. En el entendido que el proceso de aprendizaje es un proceso activo por parte de los 
Tabla 1. Validez ecológica habilidades, valores y actitudes interculturales.

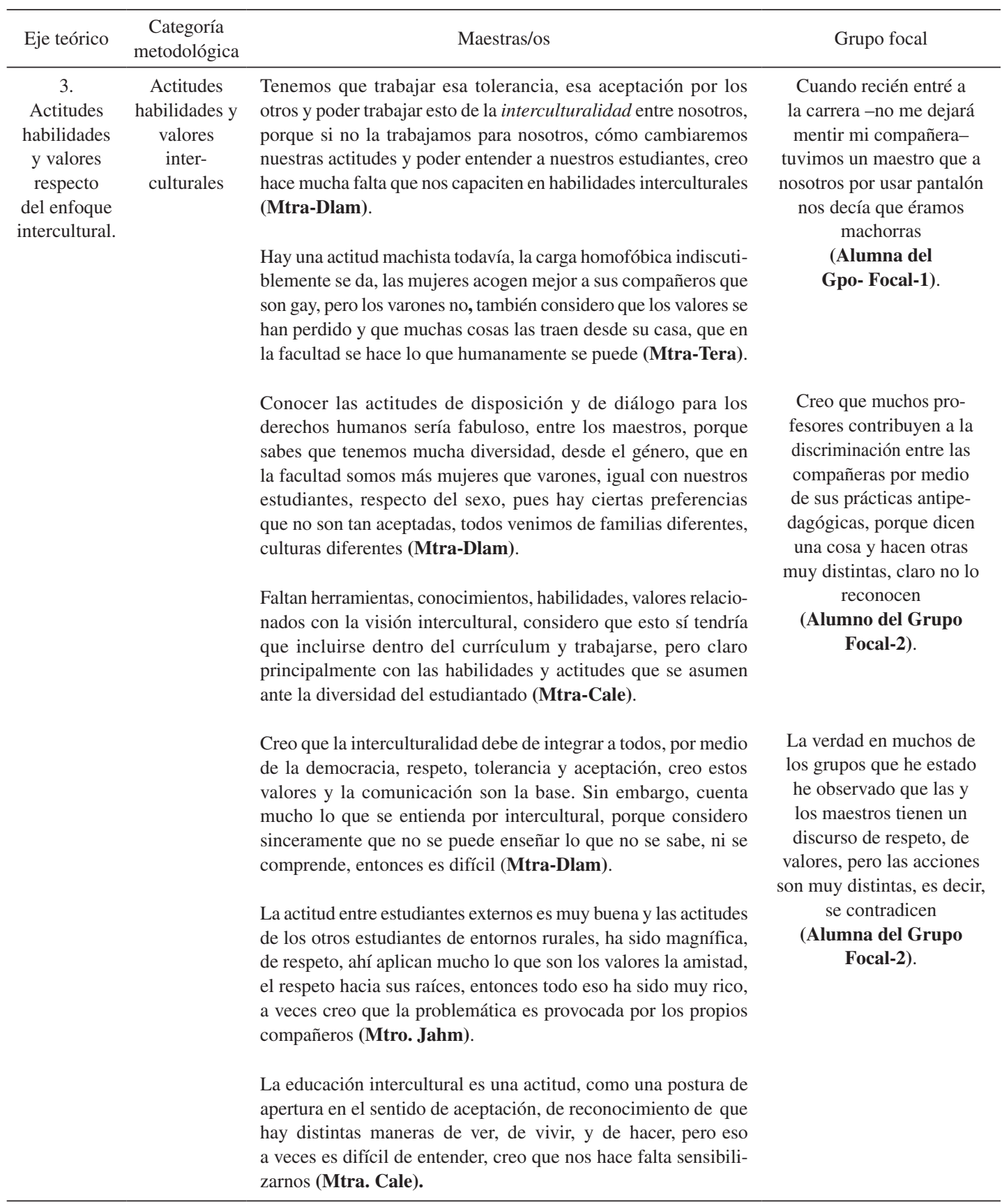

que aprenden y que tiene lugar en un contexto social, que traslada a las personas por encima de los componentes que les condicionan como seres humanos, señala Freyre (1998) "[...] esta situación constituye la capacidad de los estudiantes de aprender a aprender". Es importante significar la apertura que muestra el profesorado al reconocer la importancia de trabajar el enfoque intercultural, por el bien de las relaciones entre sus compañeros y de los estudiantes.

Lo que evidentemente es preocupante son las actitudes del profesorado cuando intentan invisibilizar 
las diferencias culturales como forma de entender la igualdad de oportunidades. Un primer paso sería reconocer como docentes que las actitudes atribuidas a estereotipos impiden alcanzar una relación de convivencia y respeto con el estudiantado. Es notable la participación e intervención en los distintos espacios educativos, con actitud crítica, reflexiva, colaborativa, ética e innovadora, la gran mayoría de los docentes han asumido su desempeño profesional con compromiso social.

En relación con su formación las y los maestros están conscientes de que las Sociedades Multiculturales nos exigen el reto educativo de encontrar los valores mínimos, consensuados, que permitan el diálogo y la igualdad entre seres humanos y el cambio de actitudes que favorezca el respeto a la diferencia y la diversidad cultural y promueva la convivencia pacífica entre los diferentes, así lo señalan (Sales y García López, 1997).

Cuando se les preguntó a los profesores ¿Consideras que la práctica docente, se ajusta o no, al enfoque intercultural? se puede apreciar que contestaron un poco de todo, pero muy vago en relación con el enfoque educativo intercultural.

Para estos efectos se presentan en la Tabla 2 las respuestas de los distintos actores sociales.

Al observar las respuestas del profesorado y de los estudiantes en relación con lo que consideran intercultural, y si lo reconocen en el ejercicio de su práctica docente, se detecta que algunos tienen muy claro la importancia del enfoque y la necesidad que existe principalmente en el profesorado universitario para abordarlo, desde sus planeaciones, estrategias y formas de intervención en la comunidad universitaria.

En el caso de otros profesores al referirse al enfoque intercultural, se coincide con Dietz (2012), se ubica como un "recurso" a ser gestionado de forma institucional mediante un diseño curricular estratégico, incluyendo los diferentes planes y programas de estudio y fundamentalmente en la formación del personal académico para abordar y gestionar en la Universidad. Señala este autor: "[...] no hablamos de diferencia en el sentido de distinguir sus fuentes y características, sino de diversidad para enfatizar la interrelación, el traslape y los cruces de las variaciones existentes" (p. 26).

La idea es entender que el tipo de sociedad multicultural ofrece la posibilidad de diseñar, crear, imaginar formas para el desarrollo de competencias interculturales en el profesorado universitario, con el reconocimiento de la diversidad humana como riqueza para favorecer el proceso de comunicación, interacción, diálogos y la generación de aprendizajes significativos en la comunidad universitaria.

\section{Conclusiones}

La experiencia docente con la que cuenta el profesorado universitario es incuestionable, la reflejan en su gran mayoría tanto maestras como maestros, la toma de decisiones que les permiten solucionar problemas de su práctica docente. Se detectó que el profesorado de la facultad de pedagogía tiene claros los objetivos curriculares del plan de estudios de Pedagogía 2000, y compartieron que se promueven en el estudiantado la capacidad para la intervención en los campos emergentes del quehacer pedagógico, profesional, ejerciendo autonomía intelectual con base en los fundamentos teóricos de la disciplina pedagógica.

Se reconoce en el profesorado universitario que efectivamente hay mucha calidad en el ejercicio de prácticas pedagógicas, sin embargo todo lo que se realiza es desde el punto de vista academicista y reflejan en su quehacer docente una cultura escolar bastante individualizada, poco proclive al trabajo colegiado. Es decir, mencionan: "mis proyectos", "mi experiencia educativa", "mis intereses educativos y personales", sin conectar con los otros, con los demás y principalmente fuera del dinamismo cultural y social que nos circunda.

Considero que se requiere, como elemento fundamental, un profesorado abierto a la comunicación, al intercambio de ideas, a los procesos formativos, aprendizajes mutuos, que les sirvan para moverse en los contextos cotidianos, para ello se necesita compartir tanto expectativas de logro, como memoria histórica, y ello representa una de las grandes dificultades dentro del diálogo intercultural y uno de los grandes límites que el currículo tiene en cuanto a interculturalidad (Sagastizabal, Perlo, Piretta y Ángeles, 2006).

Esto significa que el profesorado universitario deberá transitar a escenarios comunes para encontrar puntos de encuentro hacia un proceso dialógico que les permita reflexionar, analizar, replantear formas, actitudes, comportamientos de manera crítica acerca de cómo se construyen las diferencias y qué valor se les confiere en el contexto socioeducativo.

Se pudo observar que el profesorado universitario reconoce que hace falta incorporar en su vida institucional acciones que reconstruyan sus planes y 
Tabla 2. Validez ecológica Práctica Docente y enfoque intercultural.

\begin{tabular}{|c|c|c|c|}
\hline Eje teórico & $\begin{array}{l}\text { Categoría } \\
\text { metodológica }\end{array}$ & Maestras/os & Grupo focal \\
\hline $\begin{array}{c}\text { Práctica } \\
\text { docente } \\
\text { y enfoque } \\
\text { educativo } \\
\text { intercultural }\end{array}$ & $\begin{array}{c}\text { Prácticas } \\
\text { pedagógicas que } \\
\text { consideran, se } \\
\text { ajustan o no, } \\
\text { al enfoque } \\
\text { intercultural }\end{array}$ & $\begin{array}{l}\text { La comunicación, apertura al diálogo, desafortunadamente } \\
\text { a veces no se los permitimos a los estudiantes y entre nosotros } \\
\text { como compañeros es muy difícil también que se dé, creo eso } \\
\text { es intercultural y en eso tendríamos que trabajar más, pero } \\
\text { sobre todo nosotros (M-Dlam). } \\
\text { Intercultural podría ser, no querer cambiar al otro sino aceptarlo } \\
\text { es un poco, así como, con tu pareja, te acepto como eres, tal } \\
\text { cual, sin querer cambiar, nosotros tenemos estudiantes exce- } \\
\text { lentes que vienen de diferentes estratos y culturas totalmente } \\
\text { diferentes y eso es lo que en determinado momento tenemos que } \\
\text { aprovechar, porque al final aprendes con ellos (Mtra-Dlam). } \\
\text { La práctica depende de cada exp. educ., horarios, espacios, } \\
\text { estrategias, identificar el grupo. Según el modelo flexible, } \\
\text { pues nos llegan de distintos semestres, también debes ajustar } \\
\text { las técnicas que vas a utilizar, y ya si nos ponemos muy ins- } \\
\text { titucionales, pues competencias, proyecto aula, plataforma } \\
\text { Eminus y fomentar los tres ejes, pensamiento complejo, nuevas } \\
\text { tecnologías, investigación, entonces pues la práctica docente, } \\
\text { depende de varias cosas (Mtra-Gisp). } \\
\text { Considero que a veces los maestros, el concepto de intercultu- } \\
\text { ralidad y lo asociamos a comunidades indígenas por ejemplo } \\
\text { y no creo que sea así, nada más, creo que lo intercultural está } \\
\text { presente en todo lo que hacen los estudiantes y maestros, pero que } \\
\text { sí, sí deberíamos de saber más sobre ese modelo (Mtro-Joch). } \\
\text { Pienso que la labor del docente no es solo la transmisión de } \\
\text { conocimientos únicamente, sino la interacción humana, por } \\
\text { lo que considero sería excelente que conociéramos más al } \\
\text { respecto de los Derechos Humanos (Mtra-Gisp). }\end{array}$ & $\begin{array}{l}\text { Algunos maestros } \\
\text { trabajan y ya, pero no } \\
\text { saben de dónde eres, ni } \\
\text { de dónde vienes, yo, por } \\
\text { ejemplo, soy foránea } \\
\text { y debo viajar a diario, } \\
\text { algunos maestros no } \\
\text { lo saben, otros no se } \\
\text { aprenden ni los nombres } \\
\text { y creo deberían ir más } \\
\text { allá de lo que ven } \\
\text { (Gpo-Focal-1). } \\
\text { Considero que el pro- } \\
\text { fesorado de la facultad } \\
\text { tiene buenas intenciones } \\
\text { al querer ejercer una } \\
\text { práctica de calidad, pero } \\
\text { lo cierto es que ignoran } \\
\text { cosas como lo inter- } \\
\text { cultural, que considero } \\
\text { deberían de dominar } \\
\text { (Gpo-Focal-2). }\end{array}$ \\
\hline
\end{tabular}

programas de estudio y resignifiquen contenidos en el campo de lo intercultural. Identifican los valores y principios del enfoque intercultural, pero representa un verdadero reto reconocerlo para aplicarlo en su práctica docente. Es menester reconocer que la enseñanza-aprendizaje de actitudes y valores interculturales tienen un fuerte impacto social, pues permite desplegar voluntades para actuar de forma respetuosa ante sociedades en constante y acelerado cambio cultural, donde los conocimientos y las conductas y ciertos comportamientos son eminentemente fugaces.

Una alternativa viable sería la formación de actitudes, habilidades y valores interculturales en los profesionales de la educación, mover a la reflexión para sensibilizar y mirar desde la perspectiva intercultural con la idea de propiciar una convivencia social, en la que tengan cabida todas las diferencias y prácticas socioculturales, pero sobre la base de la aceptación de un código universal de valores y un escrupuloso respeto a la libertad, igualdad, justicia social, dignidad y profundo respeto por los derechos humanos. Un asunto importante que cuidar es, no considerar que el enfoque intercultural vendrá a resolver los problemas actuales, no es posible suponer el dar recetas en la educación, se pretende que sea un conjunto de provocaciones, que lleven al diálogo, a la interacción entre las distintas partes y agentes que participan en el proceso educativo.

Un aspecto clave es colocar al centro del debate las formas de relación que se producen entre las y los maestros, insistir que esto favorece en gran medida el ambiente en el aula y en los distintos espacios de la universidad. Así como el resto de las dimensiones de la educación y los procesos en los que el conocimiento se construye y legitima. 
Sin olvidar que el enfoque intercultural se propone como una perspectiva para definir y responder a la diversidad en la educación.

Por todo lo anterior, considero que la Universidad Veracruzana debe incorporar a sus agendas institucionales, entre otros temas de interés público, el relacionado con la formación del profesorado en el enfoque educativo intercultural, toda vez que es un espacio privilegiado de formación desde el que se pueden reproducir de manera exponencial conocimientos, habilidades, valores y actitudes que favorezcan el reconocimiento de la diverdad entre los hombres y las mujeres. En tal sentido, como interés fundamental para la sociedad en general y por tanto para las distintas instituciones educativas del estado de Veracruz y del país.

\section{Referencias Citadas}

Abdallah-Pretceille, M.

2001 La educación intercultural. Barcelona, Idea-Books.

Abdallah-Pretceille, $M$.

2006 El paradigma intercultural como mirada hacia la diversidad. ActasCongreso INTER. Madrid. Servicio Publicaciones UNED. Intercultural Recuperado de http:// www.uned.es/congreso-inter-educacion-intercultural

Aguado, T.; Gil-Jaurena, I. \& Benito, P. M.

2005 Educación intercultural: una propuesta para la transformación de la escuela (Vol. 10). Los Libros de la Catarata. Aguado, T.

2009 "El enfoque intercultural como metáfora de la diversidad en educación". Educación Intercultural perspectivas y propuestas, 13-29.

Alonso, R. S.

2006 "La educación intercultural". Revista de educación, 339, 859-881.

Berger, P.; Huntington, S. et al.

2002 Globalizaciones múltiples. La diversidad cultural en el mundo contemporáneo. Barcelona: Paidós.

Bello, D.; Aguilar, B. y Cols.

2016 La Educación Inclusiva reflexiones desde Latinoamérica. México: Castellanos editores.

Coleman, J. S.

1990 Foundations of Social Theory. Cambridge, Belknap Press of Harvard University Press.

Dietz, G.

2012 Multiculturalismo, interculturalidad y diversidad en educación. Una aproximación antropológica. México: FCE.

Dietz, G. y Mateos, L.

2009 "El discurso intercultural ante el paradigma de la diversidad", en Aguado, T., Del Olmo, M. Educación Intercultural. Perspectivas y propuestas. Ed. Interalfa.

Fernández Enguita, M.

1999 Alumnos gitanos en la escuela paya. Un estudio sobre las relaciones étnicas en el sistema educativo. Barcelona: Ariel.

Freire, $\mathrm{P}$.

1998 La educación como práctica de la libertad. Siglo XXI: Tierra Nueva.

Galeano, E.

2005 Patas arriba: la escuela del mundo al revés. Siglo XXI: Madrid.

Gómez Zermeño, M. G.

2009 Estudio Exploratorio-Descriptivo de Competencias Interculturales en Instructores Comunitarios del Consejo Nacional de Fomento Educativo que Brindan Servicio en la Modalidad de Atención Educativa a Población Indígena del Estado de Chiapas-Edición Única.
Gorski, P. C.

2000a A working definition of multicultural education, en Multicultural Pavilion. Disponible en www.edchange.org/ multicultural/initial.html. Cons. 28-2-2014.

Gimeno y cols.

1998 Maestros: formación, práctica y transformación escolar.

Hernández, R.; Fernández, C. \& Baptista, P.

2012 Metodología de la investigación. 5a. Ed. México D.F.: McGraw-Hill.

Miguélez, M.

2011 Ciencia y arte en la metodología cualitativa. Editores: México, DF, 2006.

Meneses, G.

2004 NTIC, Interacción y aprendizaje en la Universidad. Trabajo de investigación para obtener el DEA. Tarragona: Universitat Rovira I Virgili.

Navarro Sada, A. \& Maldonado, A.

2007 "Research Methods in Education", by Louis Cohen, Lawrence Manion and Keith Morrison. British Journal of Educational Studies, 55(4), 469-470. Recuperado de internet en Nov-17 de 2017.

OCDE

2009 Informe Talis, I. La creación de entornos eficaces de enseñanza y aprendizaje-Síntesis de los primeros resultados.

Sagastizabal, M.; Perlo, C.; Piretta B., San Martín y P. de los Ángeles

2006 Aprender y enseñar en contextos complejos: Multiculturalidad, diversidad y fragmentación. Centro de Publicaciones Educativas y material didáctico, Buenos Aires, Argentina.

Sales, A. y García López, R.

1997 Programas de educación intercultural. Bilbao: Desclée de Brouwer.

Soneira

2012 Bases de la investigación cualitativa: técnicas y procedimientos para desarrollar la teoría fundamentada. Medellín: Universidad de Antioquia.

Universidad Veracruzana. Plan de estudios de Pedagogía 2000 Recuperado de https://www.uv.mx/pedagogia/general/ plan-de-estudios-2/ 27/02/2018

Valles, M. S.

2000 La grounded theory y el análisis cualitativo asistido por ordenador. El análisis de la realidad social. Métodos y técnicas de investigación, 575-604.

Walsh, C.

2005 "Introducción. (Re) pensamiento crítico y (de) colonialidad". Pensamiento crítico y matriz (de) colonial, 13-36. 
